\title{
Detection and Parameter Estimation of Multicomponent LFM Signal based on Nonlinear Transformation under the Impulsive Noise
}

\section{Li Li ( $\sim$ ffsimple@163.com )}

Dalian University https://orcid.org/0000-0002-4680-0922

Xiaofei Shi

Dalian Maritime University

Mingyan $\mathrm{He}$

Dalian University

\section{Research Article}

Keywords: Internet of Vehicles, Alpha stable distribution noise, fractional Fourier domain, Tuneable Sigmoid transform, multicomponent LFM signal

Posted Date: January 27th, 2022

DOI: https://doi.org/10.21203/rs.3.rs-1287977/v1

License: (c) (i) This work is licensed under a Creative Commons Attribution 4.0 International License. Read Full License 
Research Article

\title{
Detection and Parameter Estimation of Multicomponent LFM Signal based on Nonlinear Transformation under the Impulsive Noise
}

\author{
$\mathrm{Li} \mathrm{Li}^{1}$, Xiaofei Shi ${ }^{2}$ and Mingyan $\mathrm{He}^{1}$ \\ 1 College of Information Engineering, Dalian University, Dalian, Liaoning, China \\ 2 Information Science and Technology College, Dalian maritime University, Dalian, Liaoning ,China \\ Correspondence should be addressed to Li Li, ffsimple@163.com
}

\begin{abstract}
:
The linear frequency modulated (LFM) signal has been widely implemented in broadband wireless communications in high-speed vehicles, such as internet of vehicles(IoV), because of its excellent characteristic of long time interval and wide frequency band. In this paper, a novel method, which employs the fractional Fourier transform and the Tuneable-Sigmoid transform, is proposed to estimate parameters of multicomponent LFM signals in Internet of Vehicles(IoV) under the impulsive noise environment. For the optimization in the fractional Fourier domain, an algorithm based on peak searching is proposed. And for multicomponent signals, we further propose a signal separation technique in the fractional Fourier domain which can effectively suppress the interferences on the detection of the weak components brought by the stronger components, and estimate parameters of LFM signals. Moreover, boundedness and the complexity analysis of Tuneable-Sigmoid-FFRT to the $S \alpha S$ noise are presented to evaluate the performance of the proposed method. In additional, the Cramér-Rao bound for parameter estimation is derived and computed in closed form, which shows that better performance has been achieved. Both theoretical analysis and simulations demonstrate the superior performances of the proposed approach over other existing methods.
\end{abstract}

Keywords: Internet of Vehicles, Alpha stable distribution noise, fractional Fourier domain, Tuneable Sigmoid transform, multicomponent LFM signal

Corresponding author: Li Li, Ph.D.

Affiliation: Information Engineering College, Dalian University, Dalian 116622, China

Email: ffsimple@163.com 


\section{Introduction}

Early the linear frequency modulated (LFM) signal has been widely employed in radar signal processing[1-4], especially in target detection, tracking, location and radar imaging fields, for its excellent characteristic of long time interval and wide frequency band. In recent years, wireless sensor networks, as well as military ad hoc network, have the requirement of integration with self-localization, sensing, and communication functions[5-9]. At present, broadband wireless communications in high-speed vehicles, such as aircraft and high-speed trains, are much in demand. Thus, the detection and parameter estimation of LFM signal is an attractive topic in internet of vehicles(IoV)[10-11]. Various algorithms have been proposed to estimate the LFM signal in the Gaussian noise environment[12-15]. For instance, Ahmet Serbes proposed analytical formulations, approximations, upper and lower bounds for the angle sweep of maximum magnitude of fractional Fourier transform of mono- and multicomponent LFM signals. It employs a successive coarse-to-fine grid-search algorithm to estimate the chirp rates of multicomponent nonseparable LFM signals[12]. Aldimashki et al. proposed a fast and powerful method for the estimation of chirp rates in the fractional Fourier domains based on the golden section search[13]. Bai et al. proposed a new chirp rate estimation algorithm by multiple discrete polynomial phase transform (DPT) and weighted combination[14]. Guo et al. proposed two constraints of time-modulation frequency based on time-modulation principle, as well as two conditions of time schemes are drawn according to the law of Nyquist sampling[15]. Studies and experimental measurements showed that the IoV signal may contain impulse noise that are non-Gaussian, primarily owing to impulsive phenomena [16-20]. It is inappropriate to model the noise as Gaussian noise.

To reduce the impulsive noise interference, many parameter estimation algorithms were proposed, especially, based on fractional lower order statistics (FLOS) [18-21]. According to the fractional lower order statistics theory, the methods based on fractional lower order statistics depend on a priori knowledge of the noise. Therefore, the performance of these algorithms based on FLOS may degrade seriously for an inappropriate fractional lower order moment.

Time-frequency distribution is a useful tool to extract helpful information of the LFM signal[21-24]. Various time frequency distribution methods are proposed, such as fractional Fourier transform (FRFT), short time Fourier transform and Wigner-Ville distributions. The Sigmoid function is widely used as a common nonlinear transform. The Sigmoid function can suppress impulsive noise interference, and this does not depend on a priori knowledge of the noise[25-29].

To handle this problem, a novel time frequency distribution based on Tuneable Sigmoid transform, referred to as fractional Fourier transform based on tuneable Sigmoid (TS-FRFT), is proposed to estimate parameters of multicomponent LFM signals under the impulsive noise environment, without the requirement of the priori knowledge about impulsive noise.

This paper is organized as follows. In section 2, performance analysis of fractional Fourier transform fractional Fourier transform is presented. In section 3, a novel parameters estimation method based on TS-FRFT for impulsive noise is proposed. In section 4, the performance of the TS-FRFT method is analyzed. In section 5, the performance of the parameter estimation algorithm is studied through extensive numerical simulations. Finally, conclusions are drawn in section 6.

\section{Method}

\subsection{Performance analysis of fractional Fourier transform}

\subsubsection{Fractional Fourier transform}

The fractional Fourier transform (FRFT) is a generalization of the FT and can be interpreted as a rotation of the signal to any angles in the time-frequency plane [23-24].

The continuous FRFT of a signal $x(t)$ with a rotation angle $\rho$ is defined as 


$$
X(\rho, m)=F^{\rho}[x(t)](m)=\int_{-\infty}^{+\infty} x(t) K_{b}(t, m) \mathrm{d} t
$$

where $F^{\rho}$ denotes the FRFT operator, $b(0<b \leq 2)$ denotes the fractional order, $\rho \equiv b \pi / 2$, and $K_{b}(t, m)$ is the kernel function of the fractional Fourier transform. $K_{b}(t, m)$ can be expressed as

$$
K_{b}(t, m)= \begin{cases}A_{\rho} \exp \left(\frac{j}{2}\left(t^{2} \cot \rho-2 m t \csc \rho+m^{2} \cot \rho\right)\right), & \rho \neq n \pi \\ \delta(t-m), & \rho=2 n \pi \\ \delta(t+m), & \rho=(2 n+1) \pi\end{cases}
$$

where $A_{\rho}=\sqrt{\frac{1-j \cot \rho}{2 \pi}}, \rho$ denotes rotation angle in FRFT domain, $m$ denotes the frequency in FRFT domain, and $F^{\rho}[\cdot](m)$ denotes the fractional Fourier transform.

\subsubsection{Parameter estimation based on fractional Fourier transform in the Gaussian noise}

Assume that the LFM signal $x(t)$ with Gaussian noise is modeled as

$$
x(t)=s(t)+w(t)
$$

where $s(t)=\exp \left(j 2 \pi\left(f_{0} t+\mu_{0} t^{2} / 2\right)\right), \quad f_{0}$ denotes the initial frequency, $\mu_{0}$ denotes chirp rate, and $w(t)$ denotes the Gaussian noise with zero-mean.

According to (1) and (3), the FRFT of the LFM signal $x(t)$ can be expressed as,

$$
\begin{aligned}
X(\rho, m) & =F^{b}[x(t)](m) \\
& =A_{\rho} \exp \left(j \pi m^{2} \cot \rho\right) \cdot \int_{-T / 2}^{-T / 2} \exp \left(j 2 \pi t\left(f_{0}-m \csc \rho\right)\right) \exp \left(j \pi t^{2}\left(\cot \rho+\mu_{0}\right)\right) \mathrm{d} t
\end{aligned}
$$

When $\mu_{0}=-\cot \rho, X(\rho, m)$ has the best energy- concentrated property and an optimal rotation angle $\rho_{0}$ exists to maximize the peak amplitude of $X(\rho, m)$. Accordingly, $b_{0}=2 \rho_{0} / \pi$ is called the optimal fractional order. The $X\left(\rho_{0}, m\right)$ forms a pulse in the FRFT domain and its peak value appears at $\left(\rho_{0}, m_{0}\right)$ as

$$
\left\{\begin{array}{l}
\cot \rho_{0}=-\mu_{0} \\
m_{0} \csc \rho_{0}=f_{0} \\
\left(\rho_{0}=-\operatorname{arccot} \mu_{0}, m_{0}=f_{0} \sin \rho_{0}\right)=\underset{\rho, m}{\arg \max }|X(\rho, m)|
\end{array}\right.
$$

Then, parameters $f_{0}$ and $\mu_{0}$ could be estimated directly from (6) by

$$
\left\{\begin{array}{l}
\mu_{0}=-\cot \rho_{0} \\
f_{0}=m_{0} \csc \rho_{0}
\end{array}\right.
$$

\subsubsection{The $S \alpha S$ Distribution Noise}

In this section, we introduce the statistical model that will be used to describe the additive noise. The model is based on the class of bivariate symmetric $\alpha$-stable ( $S \alpha S$ ) distribution and is well-suited for describing noise processes that are impulsive in nature. Its characteristic function of the $S \alpha S$ distribution is defined as[17-19]:

$$
\psi(\omega)=\exp \left(-\gamma|\omega|^{\alpha}\right)
$$

where the parameter $\alpha$ is usually called the characteristic exponent. It can be proven that, in order for (7) to define a characteristic function, the values of $\alpha$ must be restricted to the interval $0<\alpha \leq 2$. When $0<\alpha<2$, the distribution is 
algebraic tailed with tail constant $\alpha$, implying infinite variance. When $\alpha=2$, the distribution is Gaussian, implying lighterthan-algebraic tails.

The parameter $\gamma$, usually called the dispersion, is a positive constant related to the scale of the distribution. For a fixed $\alpha$, larger values of $\gamma$ correspond to larger strengths of the process. It is easy to see that $\gamma^{1 / \alpha}$ is, in fact, a scale parameter of the distribution.

\subsubsection{Performance analysis of FRFT to the $S \alpha S$ noise}

We consider $x(t)$ as an observed signal, defined as

$$
x(t)=s(t)+n(t)
$$

where $s(t)$ denotes the LFM signal and the noise $n(t)$ is a sequence of i.i.d isotropic complex $S \alpha S$ random variable.

The fractional Fourier power spectrum function is defined as[23]

$$
P_{x x}^{\rho}(m)=\lim _{T \rightarrow \infty} \frac{E|X(\rho, m)|^{2}}{2 T}
$$

where $X(\rho, m)$ is the FRFT of the signal $x(t)$. Then, substituting (8) into (9), we get

$$
\begin{aligned}
P_{x x}^{\rho}(m) & =\lim _{T \rightarrow \infty} \frac{1}{2 T} E\left[\int_{-\mathrm{T}}^{+T} x\left(t_{1}\right) K_{\rho}\left(t_{1}, m\right) \mathrm{d} t_{1} \int_{-\mathrm{T}}^{+T} x^{*}\left(t_{2}\right) K_{\rho}^{*}\left(t_{2}, m\right) \mathrm{d} t_{2}\right] \\
& =\lim _{T \rightarrow \infty} \frac{1}{2 T} E\left[\int_{-\mathrm{T}}^{+T} \int_{-\mathrm{T}}^{+T} K_{\rho}\left(t_{1}, m\right) K_{\rho}^{*}\left(t_{2}, m\right) x^{*}\left(t_{2}\right) x\left(t_{1}\right) \mathrm{d} t_{1} \mathrm{~d} t_{2}\right] \\
& =\lim _{T \rightarrow \infty} \frac{1}{2 T} \int_{-\mathrm{T}}^{+T} \int_{-\mathrm{T}}^{+T} K_{\rho}\left(t_{1}, m\right) K_{\rho}^{*}\left(t_{2}, m\right) E\left[x\left(t_{1}\right) x^{*}\left(t_{2}\right)\right] \mathrm{d} t_{1} \mathrm{~d} t_{2}
\end{aligned}
$$

where $x\left(t_{1}\right)$ and $x\left(t_{2}\right)$ are the sample values of $x(t)$.

According to the properties of $\alpha$-stable distribution, if the signal $x(t)$ contains the $S \alpha S$ distribution noise, then $x(t)$ does not have finite second-order moments. Therefore, $E\left[x\left(t_{1}\right) x^{*}\left(t_{2}\right)\right]$ is unbounded for $S \alpha S$ process, i.e., $P_{x x}^{\rho}(m)$ is unbounded for $S \alpha S$ stable distribution noise. Thus, the algorithm based on the FRFT becomes unbounded when the signal contains alpha-stable distribution noise, and this peak location algorithm may fail.

Therefore, we present a nonlinear transform, tuneable- Sigmoid transform, to suppress the alpha-stable distribution noise interference.

\subsection{Parameters estimation based on Tuneable- Sigmoid-FRFT}

\subsubsection{Tuneable Sigmoid transform}

In fact, many nonlinear functions can be used to suppress the lower order $\alpha$-stable noise. The ideal nonlinear transform function should have following features: it can eliminate the impact of the impulsive noises, but it does not cause a severe distortion to the normal LFM signal. The tuneable-Sigmoid function widely used in the artificial neural network is a very good nonlinear function for both purposes. By using the tuneable Sigmoid function[25], this paper proposes a nonlinear transform-based parameter estimation algorithm.

The nonlinear transform, tuneable Sigmoid function, is defined as

$$
T S[x(t)]=\frac{2}{1+\exp [-\lambda x(t)]}-1
$$

where $\lambda$ is used as a scale factor to fit various signals and noises. 


\subsubsection{Definition of Tuneable-Sigmoid-FRFT}

In section, a novel tuneable Sigmoid fractional Fourier transform (TS-FRFT), combining the fractional Fourier transform and the tuneable Sigmoid transform is proposed to suppress the interference of the impulsive noise. The definition of the tuneable-Sigmoid-FRFT $\quad X_{T S}(\rho, m)$ is expressed as

$$
\begin{aligned}
X_{T S}(\rho, m) & =F_{T S}^{b}[x(t)](m) \\
& =\int_{-\infty}^{+\infty} T S[x(t)] K_{\rho}(t, m) \mathrm{d} t
\end{aligned}
$$

Since the FRFT spectrum $X(\rho, m)$ of the LFM signal $x(t)$ has the energy-concentrated property, the tuneableSigmoid-FRFT spectrum $X_{T S}(\rho, m)$ of $T S[x(t)]$ demonstrates the same property according to Properties of the tuneableSigmoid transform [25]. Furthermore, $X(\rho, m)$ and $X_{T S}(\rho, m)$ form the pulse at the same location in the FRFT domain.

Fig.1 shows the estimation results of the FRFT and tuneable-Sigmoid-FRFT for the multi-component LFM signals under impulsive noise with GSNR $=5 \mathrm{~dB}$ and $\alpha=1.2$. From Fig.1 (a)-(b), we can find, compared with the FRFT spectrum of the LFM signal, the FRFT peak cannot be easily separated from the impulsive noise in the FRFT spectrum of the multicomponent LFM signal with impulsive noise. Thus, the correct peak cannot be obtained and the estimation performance degrades severely in the impulsive noise environment. Compared with the FRFT spectrum of the LFM signal, the tuneableSigmoid-FRFT spectrum of the LFM signal with impulsive noise forms two obvious pulses that is because the nonlinear transform restrains impulsive noise interference, as illustrated is Fig.1 (c)-(d). Therefore, the proposed method based on the tuneable- Sigmoid-FRFT can effectively suppress impulsive noise interference, yields an accurate peak estimation, and has a better estimation performance.

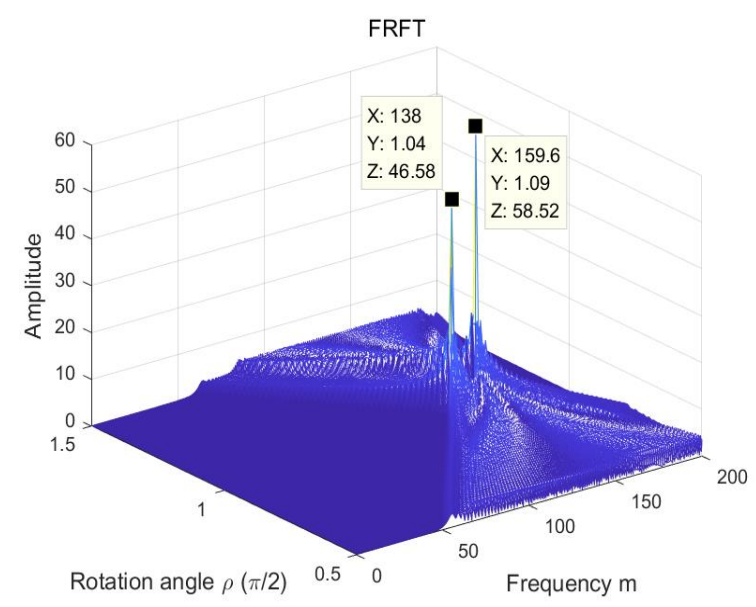

(a)

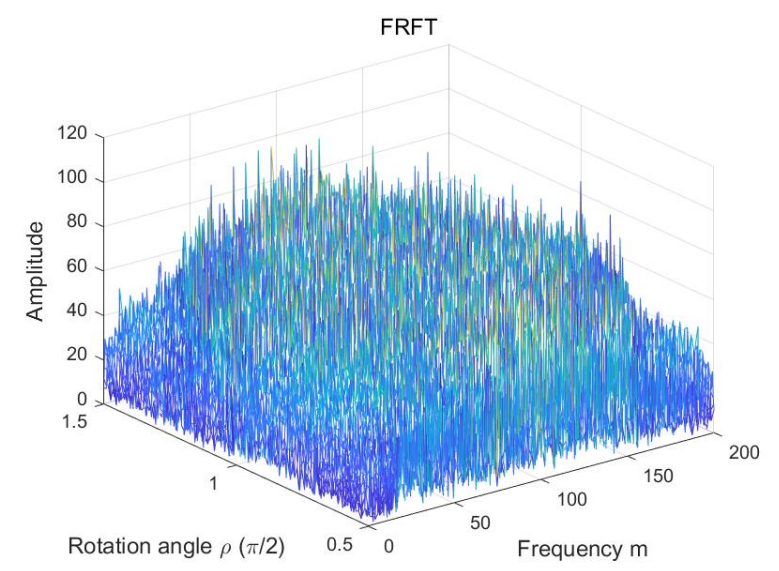

(b) 


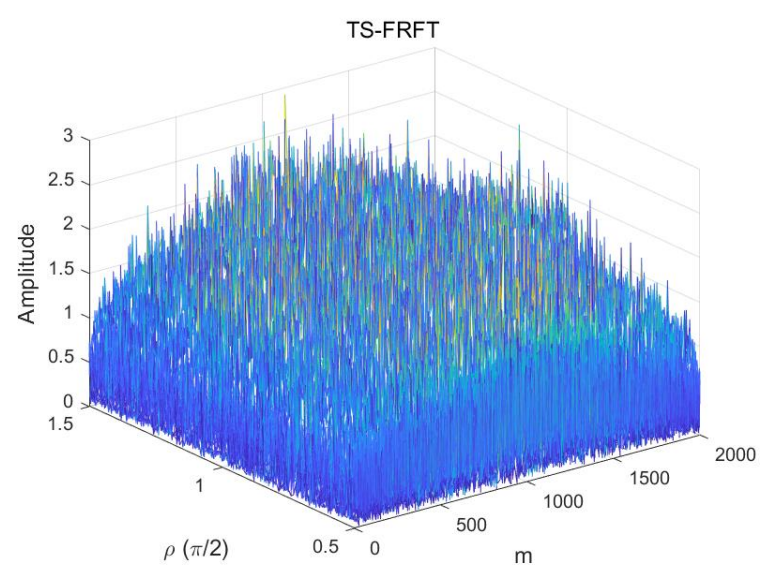

(c)

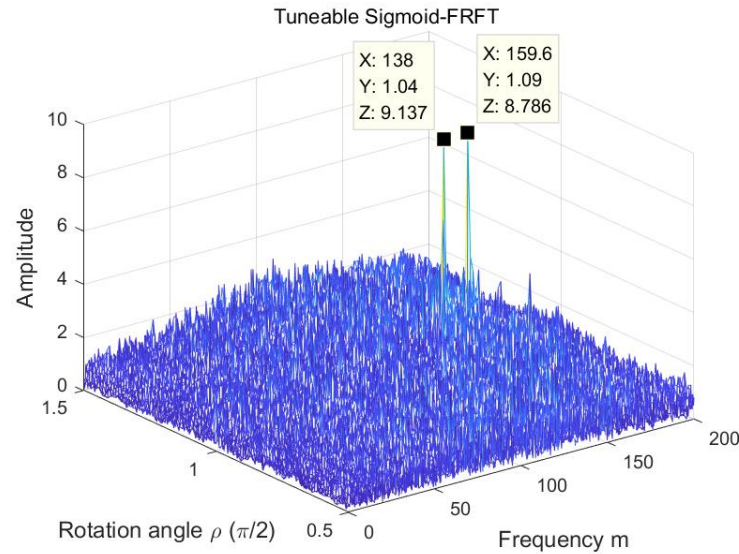

(d)

Fig. 1 The spectrum of FRFT, TS-FRFT with impulsive noise (GSNR $=5 \mathrm{~dB}$ and $\alpha=1.2$ ).(a)The FRFT spectrum of the LFM signal; (b)

The FRFT spectrum of the LFM signal with impulsive noise. (c) The TS-FRFT spectrum of the impulsive noise; (d) The TS-FRFT spectrum of the multi-component LFM signals with impulsive noise

\subsubsection{Parameters estimation of LFM Signal based on TS-FRFT}

Suppose the signal $y(t)$ with alpha-stable distribution noise as

$$
\mathrm{y}(t)=s(t)+n(t)
$$

where the noise $n(t)$ denotes a sequence of i.i.d isptropic complex $S \alpha S$ random variable.

According to (12), the tuneable-Sigmoid-FRFT of the signal $y(t)$ can be expressed as

$$
\begin{aligned}
Y_{\mathrm{TS}}(\rho, m) & =F_{\mathrm{TS}}^{b}[y(t)](m) \\
& =F_{\mathrm{TS}}^{b}\left[\exp \left(j 2 \pi\left(f_{0} t+\mu_{0} t^{2} / 2\right)\right)\right](m)+F_{\mathrm{TS}}^{b}[n(t)](m) \\
& =\int_{-\infty}^{+\infty} \mathrm{TS}\left[\exp \left(j 2 \pi\left(f_{0} t+\mu_{0} t^{2} / 2\right)\right)\right] K_{\rho}(t, m) \mathrm{d} t+N_{\mathrm{TS}}(\rho, m)
\end{aligned}
$$

where $N_{\mathrm{TS}}(\rho, m)$ denotes the tuneable-Sigmoid-FRFT of the noise $n(t)$.

By searching peak of the TS-FRFT, the chirp rate $\mu_{0}$ and initial frequency $f_{0}$ can be jointly estimated by

$$
\left\{\begin{array}{l}
\cot \rho_{0}=-\mu_{0} \\
m_{0} \csc \rho_{0}=f_{0} \\
\left(\rho_{0}=-\operatorname{arccot} \mu_{0}, m_{0}=f_{0} \sin \rho_{0}\right)=\underset{\rho, m}{\arg \max }|Y(\rho, m)|
\end{array}\right.
$$

\subsection{The analysis of the Tuneable-Sigmoid-FRFT}

\subsubsection{Boundedness of TS-FRFT to the $S \alpha S$ noise}

Assumed the signal $x(t)$ contains the impulsive noise, we can defined as

$$
x(t)=s(t)+n(t)
$$

where $s(t)$ is the signal and $n(t)$ is the $S \alpha S$ random variable noise.

The fractional power spectrum function based on TS-FRFT is defined as[23]

$$
P_{x x}^{\rho}(m)=\lim _{T \rightarrow \infty} \frac{E\left|X_{\mathrm{TS}}(\rho, m)\right|^{2}}{2 T}
$$


where $X_{\mathrm{TS}}(\rho, m)$ is the TS-FRFT of the signal $x(t)$. Then, substituting (16) into (17), we get

$$
\begin{aligned}
P_{x x}^{\rho}(m) & =\lim _{T \rightarrow \infty} \frac{1}{2 T} E\left[\int_{-\mathrm{T}}^{+T} T S\left[x\left(t_{1}\right)\right] K_{\rho}\left(t_{1}, m\right) \mathrm{d} t_{1} \int_{-\mathrm{T}}^{+T} T S^{*}\left[x\left(t_{2}\right)\right] K_{\rho}^{*}\left(t_{2}, m\right) \mathrm{d} t_{2}\right] \\
& =\lim _{T \rightarrow \infty} \frac{1}{2 T} E\left[\int_{-\mathrm{T}}^{+T} \int_{-\mathrm{T}}^{+T} K_{\rho}\left(t_{1}, m\right) K_{\rho}^{*}\left(t_{2}, m\right) T S^{*}\left[x\left(t_{2}\right)\right] T S\left[x\left(t_{1}\right)\right] \mathrm{d} t_{1} \mathrm{~d} t_{2}\right] \\
& =\lim _{T \rightarrow \infty} \frac{1}{2 T} \int_{-\mathrm{T}}^{+T} \int_{-\mathrm{T}}^{+T} K_{\rho}\left(t_{1}, m\right) K_{\rho}^{*}\left(t_{2}, m\right) E\left[T S\left[x\left(t_{1}\right)\right] T S^{*}\left[x\left(t_{2}\right)\right]\right] \mathrm{d} t_{1} \mathrm{~d} t_{2}
\end{aligned}
$$

where $x\left(t_{1}\right)$ and $x\left(t_{2}\right)$ are the sample values of $x(t)$.

According to the properties of the tuneable-Sigmoid transform, we can obtain that $E\left[T S\left[x\left(t_{1}\right)\right] T S^{*}\left[x\left(t_{2}\right)\right]\right]$ is bounded for for the $S \alpha S$ process because it is only involved with $T S[x(t)]$. Therefore, the boundedness of the $T S[x(t)]$ can guarantee the boundedness of $P_{x x}^{\beta}(m)$ under the $S \alpha S$ noise, resulting in that $X_{\mathrm{TS}}(\rho, m)$ is bounded.

\subsubsection{Complexity Analysis}

In this section, we evaluate the computational complexity of the proposed method, FRFT method, and FLOS-FPSD method. Suppose that the data length is $N$, the computational complexity of the FRFT method is $O\left(N \log _{2} N\right)[24]$. The computational complexity of the FLOS-FPSD algorithm is $O\left(N^{2}+N \log _{2} N\right)$. The proposed method based on the TS-FRFT requires two steps. The first step performs the tuneable-Sigmoid transform and the second step carries the FRFT transform. Thus, the computational complexity of the tuneable-Sigmoid-FRFT algorithm is $O\left(N+N \log _{2} N\right)$. Through the computational complexity analysis, the tuneable-Sigmoid-FRFT method can suppress impulsive noise interference and has a lower computational complexity compared with the FLOS-FPSD method.

\subsubsection{The Cramer-Rao Bound}

In this section, we derive a novel explicit expressions for the exact Cramer-Rao Bound (CRB) on the accuracy of estimating the LFM signal with impulsive noise.

The CR bound expresses a lower bound for the variance of an unbiased estimate and is, in general, not too difficult to compute[30-34]. By comparing the performance of an estimator to the CR bound, we can often have an indication on how close the estimator is to the optimum.

The LFM signal with impulsive noise can be expressed as

$$
\boldsymbol{y}(t)=\boldsymbol{x}(t)+\boldsymbol{n}(t)
$$

where $x(t)$ is the LFM signal and $n(t)$ is the $S \alpha S$ random variable noise..

The two parameters to be estimated are the chirp rate $\mu_{0}$ and initial frequency $f_{0}$, which form the parameter vector $\xi$ such that $\xi=\left[f_{0}, \mu_{0}\right]^{\mathrm{T}}$, where $[\cdot]^{\mathrm{T}}$ denotes the transpose of a vector. Suppose that the number of snapshots is $N$.

We may approximate the conditional probability density function (PDF) according to the model in (20) as

$$
p(y \mid \xi)=K \exp \left\{-\frac{1}{N} \int_{-T_{0} / 2}^{T_{0} / 2}|y(t)-x(t)|^{2} d t\right\}
$$

where $K$ is a constant, and $T_{0}$ is large enough such that $x\left(a_{l}\left(t-\tau_{l}\right)\right) \approx 0$, for $|t|>T_{0} / 2$. The CR bound for an unbiased estimate $\hat{\xi}$ of real deterministic parameters $\xi$ is given by [30]

$$
\operatorname{cov}(\hat{\xi}) \triangleq E\left[(\hat{\xi}-\xi) \cdot(\hat{\xi}-\xi)^{\mathrm{T}}\right] \geq \boldsymbol{J}(\xi)^{-1}
$$


where $\boldsymbol{J}(\xi)$ is the fisher information matrix given by

$$
\boldsymbol{J} \triangleq E\left[\nabla_{\xi}(\ln p(\mathrm{y}(t) \mid \xi)) \cdot \nabla_{\xi}^{\mathrm{T}}(\ln p(y(t) \mid \xi))\right]
$$

with

$$
\nabla_{\xi} \triangleq\left[\frac{\partial}{\partial f_{0}} \frac{\partial}{\partial \mu_{0}}\right]^{\mathrm{T}}
$$

Thus, we will obtain closed-form expressions for all particular sub-blocks of the Fisher information matrix (FIM). The element $i, j$ of the FIM for estimating the vector $\xi=\left[f_{0}, \mu_{0}\right]^{\mathrm{T}}$ can be shown as,

$$
\boldsymbol{J}_{i j}=2 \operatorname{Re} \sum_{t=1}^{N}\left\{\left(\frac{\partial \boldsymbol{x}(t)}{\partial \boldsymbol{\xi}_{i}}\right)^{H} \boldsymbol{Q}_{n}^{-1}\left(\frac{\partial \boldsymbol{x}(t)}{\partial \boldsymbol{\xi}_{j}}\right)\right\}
$$

The power of a second-order process, $E\left(X^{2}\right)$ has been widely accepted in signal processing as a standard measure of signal strength. In the especial case of heavy algebraic tails $0<\alpha \leq 2$, the second-order power is always infinite and does not give useful information about process strength. In order to develop signal processing tools for the class of logarithmicorder processes, it is necessary to present the definition of geometric power.

The geometric power of the symmetric $\alpha$-stable random variables [18] as defined in (25), is given by

$$
S_{0}=\left(C_{g} \gamma\right)^{1 / \alpha} / C_{g}
$$

where $C_{g} \approx 1.78$, is the exponential of the Euler constant.

It can be easily shown that the geometric power is a scale parameter, and as such, it can be effectively used as an indicator of process strength or 'power' in situations where second-order methods are inadequate. The geometric power $S_{0}$ is used to represent the power of symmetric $\alpha$-stable random noise, i.e., $Q_{n}=S_{0} I_{N}$.

Using (24) and (25), the following explicit expressions for the blocks of the FIM are derived as follows

$$
\boldsymbol{J}=\left[\begin{array}{ll}
\boldsymbol{J}_{f_{0} f_{0}}(\xi) & \boldsymbol{J}_{f_{0} \mu_{0}}(\xi) \\
\boldsymbol{J}_{\mu_{0} f_{0}}(\xi) & \boldsymbol{J}_{\mu_{0} \mu_{0}}(\xi)
\end{array}\right]
$$

where

$$
\begin{gathered}
\boldsymbol{J}_{f_{0} f_{0}}(\xi)=2 \operatorname{Re} \sum_{t=1}^{N}\left\{\left(\boldsymbol{x}_{f_{0}}^{\prime}(t)\right)^{H} \boldsymbol{Q}_{n}^{-1}\left(\boldsymbol{x}_{f_{0}}^{\prime}(t)\right)\right\} \\
\boldsymbol{J}_{\mu_{0} \mu_{0}}(\xi)=2 \operatorname{Re} \sum_{t=1}^{N}\left\{\left(\boldsymbol{x}_{\mu_{0}}^{\prime}(t)\right)^{H} \boldsymbol{Q}_{\mu_{0}}^{-1}\left(\boldsymbol{x}_{\tau}^{\prime}(t)\right)\right\} \\
\boldsymbol{J}_{\mu_{0} f_{0}}(\xi)=\boldsymbol{J}_{f_{0} \mu_{0}}(\xi)=2 \operatorname{Re} \sum_{t=1}^{N}\left\{\left(\boldsymbol{x}_{f_{0}}^{\prime}(t)\right)^{H} \boldsymbol{Q}_{n}^{-1}\left(\boldsymbol{x}_{\mu_{0}}^{\prime}(t)\right)\right\}
\end{gathered}
$$

where $\quad \boldsymbol{x}_{f_{0}}^{\prime}(t) \triangleq \frac{\partial \boldsymbol{x}_{f_{0}}(t)}{\partial f_{0}}$ and $\boldsymbol{x}_{\mu_{0}}^{\prime}(t) \triangleq \frac{\partial \boldsymbol{x}_{\mu_{0}}(t)}{\partial \mu_{0}}$.

The expression for the CRB for the parameters are shown as

$$
\operatorname{CRB}(\xi)=J^{-1}
$$

\section{Simulation results and discussion}


In this section, we evaluate the relative performance of the FRFT method, the FLOS-FPSD method, and the Cramer-Rao Bound under the impulsive noise, respectively.

The parameters of the multicomponent LFM signal in the simulation are assumed as follows. The initial frequency and the chirp rate of three LFM signals are set to $f_{10}=0.25 f_{s}, f_{20}=0.5 f_{s}, f_{20}=0.75 f_{s}, \mu_{10}=0.1 f_{s}^{2} / N, \mu_{20}=0.2 f_{s}^{2} / N$ and $\mu_{30}=0.4 f_{s}^{2} / N$, respectively. The sampling rate is set to $f_{s}=1 \mathrm{MHz}$ with a sampling length of $N=1000$. The numbers of Monte Carlo runs is 100 in simulation 2 and 3. Through the analysis, the inclined coefficient $\lambda$ for the TS-FRFT is set as $\lambda=1$ in all the later simulations of this paper. We use the generalized signal-noise-ratio (GSNR) [20], which is defined as:

$$
\operatorname{GSNR}=10 \log \left(\sigma_{x}^{2} / \gamma\right)
$$

where $\sigma_{x}^{2}$ and $\gamma$ are the variance of the underlying signal and dispersion of the $S \alpha S$ noise, respectively.

Simulation 1: $\quad$ FRFT and Tuneable-Sigmoid-FRFT for multi-components LFM Signals

Fig.2 shows the estimation results of the FRFT and tuneable-Sigmoid-FRFT for three LFM signals under impulsive noise with $G S N R=5 \mathrm{~dB}$ and $\alpha=1.2$. From Fig.2 (a)-(b), we can find that the FRFT algorithm fails when an impulsive occurs. Compared with the FRFT spectrum of the LFM signal, the FRFT peak cannot be easily separated from the impulsive noise in the FRFT spectrum of the LFM signal with impulsive noise. Thus, the correct peak cannot be obtained and the estimation performance degrades severely in the impulsive noise environment. Compared with the FRFT spectrum of the LFM signal, the TS-FRFT spectrum of the LFM signal with impulsive noise also forms three obvious pulses, that is because the tuneableSigmoid transform restrains impulsive noise interference, as illustrated is Fig.2 (c), the TS-FRFT of the LFM signal with impulsive noise both form three obvious pulses. Therefore, the proposed method based on the TS-FRFT can effectively suppress impulsive noise interference, yields three accurate peaks, and has a better estimation performance.
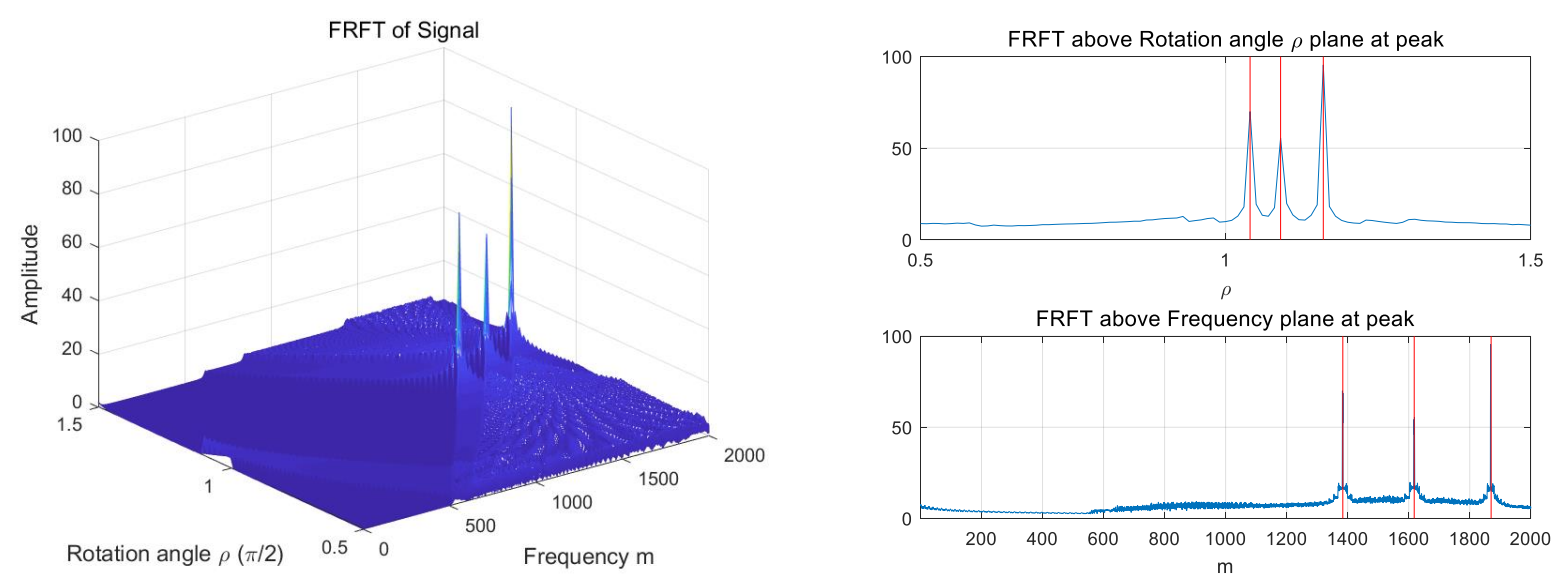

(a) 

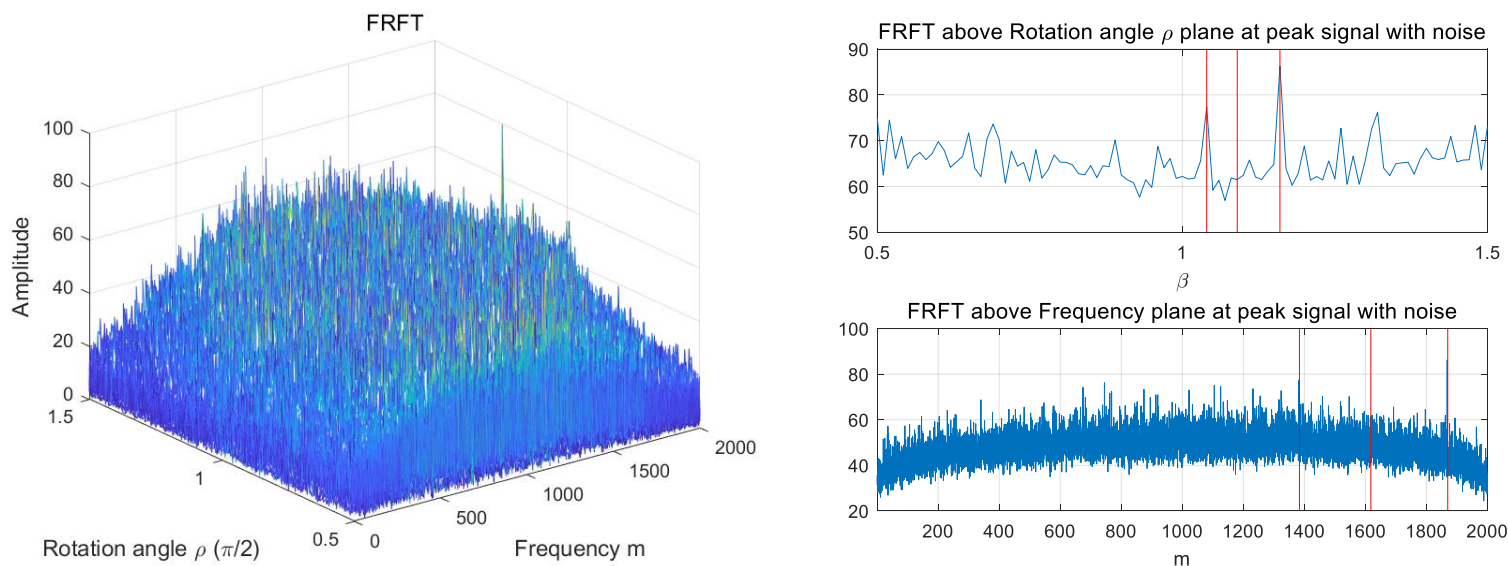

(b)
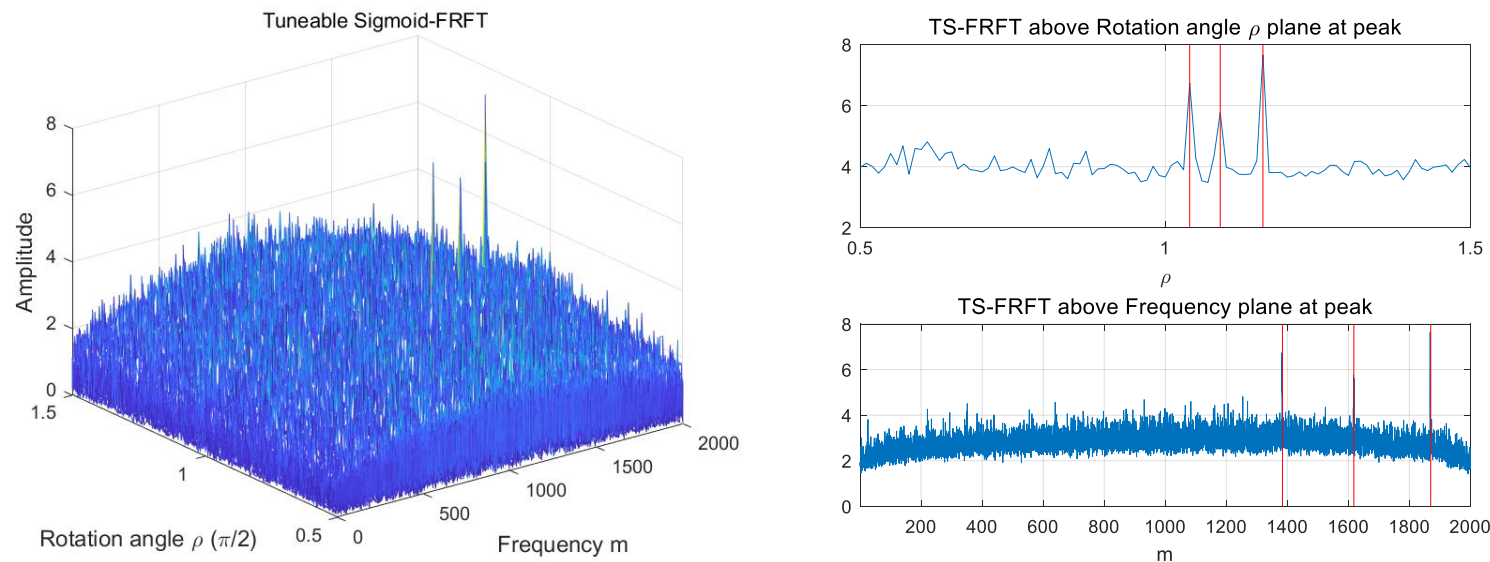

(c)

Fig.2 The spectrum of FRFT and TS-FRFT under the SaS noise. (a) The FRFT spectrum of the LFM signals without noise and its rotation angle and frequency section planes. (b) The FRFT spectrum of the LFM signals with impulsive noise and its rotation angle and frequency section planes. (c) The tuneable-Sigmoid-FRFT spectrum of the LFM signals with impulsive noise and its rotation angle frequency section planes

In Simulation 2 and Simulation 3, we compare the performance of TS-FRFT, FRFT, FLOS-FPSD and the Cramer-Rao Lower Bound (CRLB) under the impulsive noise.

\section{Simulation 2: Estimation accuracy with respect to GSNR}

To evaluate the performance of initial frequency and chirp rate, in this simulation, the characteristic exponent is set to $\alpha=1.2$ and the fractional lower order moment is set to $p=1.3$ for the FLOS-FPSD method. The resulting RMSE performance versus GSNR is illustrated in Fig.3. 


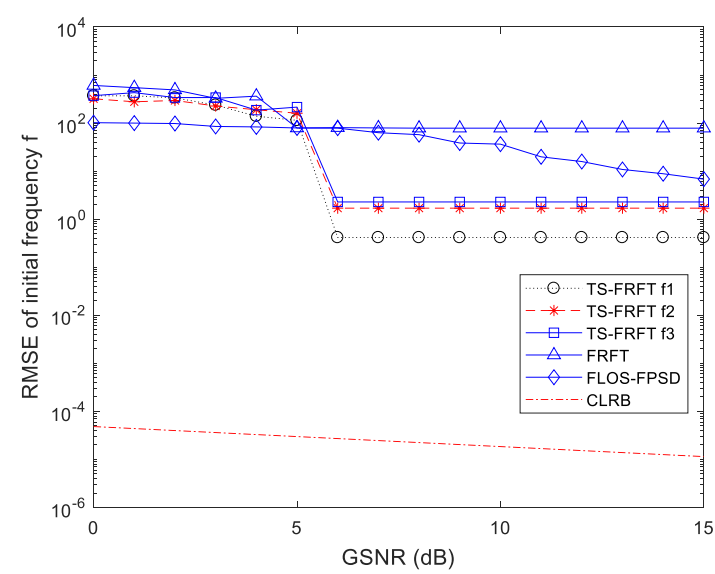

(a)

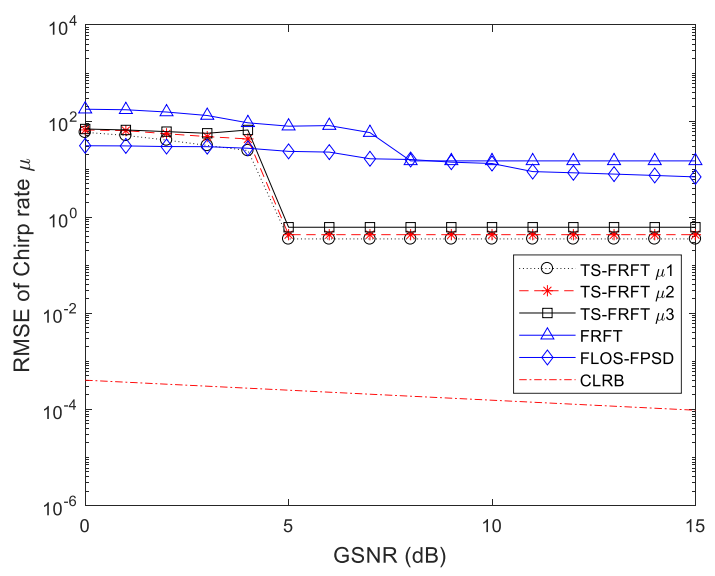

(b)

Fig.3 Estimation accuracy versus GSNR. (a) RMSE of initial frequency. (b) RMSE of Chirp rate.

From Fig.3, we can find that the FRFT method and FLOS-FPSD method both have a poor estimation performance with the $S \alpha S$ noise interference. The FLOS-FPSD method with $\alpha=1.2$ and $p=1.3$, combining the fractional lower order statistics theory with the fractional power spectrum density, can effectively suppress the $S \alpha S$ noise interference. On the contrary, the TS-FRFT method can suppress the $S \alpha S$ noise interference employing tuneable-Sigmoid transform, and the estimation performance of the TS-FRFT cannot be affected by the fractional lower-order moment $p$ value. Therefore, the performance of the TS-FRFT method outweighs those methods.

\section{Simulation 3: Estimation accuracy with respect to Characteristic Exponent $\alpha$}

In this simulation, the GSNR is set to $G S N R=5 \mathrm{~dB}$ and the fractional lower order moment is set to $p=1.1$ for the FLOS-FPSD method. Fig.4 shows the performance versus characteristic exponent $\alpha$. From Fig.4, we can find that the FPSD algorithm has a better estimation performance when the characteristic exponent $\alpha$ is close to 2. The FLOS-FPSD method may suppress $\alpha$-stable distribution noise interference employing the fractional lower order statistic theory. The performance of the FLOS-FPSD method is shown to be better than that of the FRFT method.

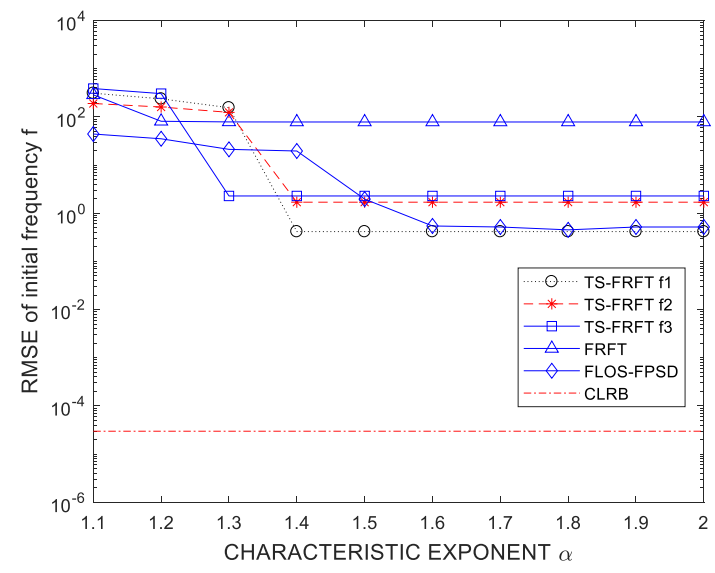

(a)

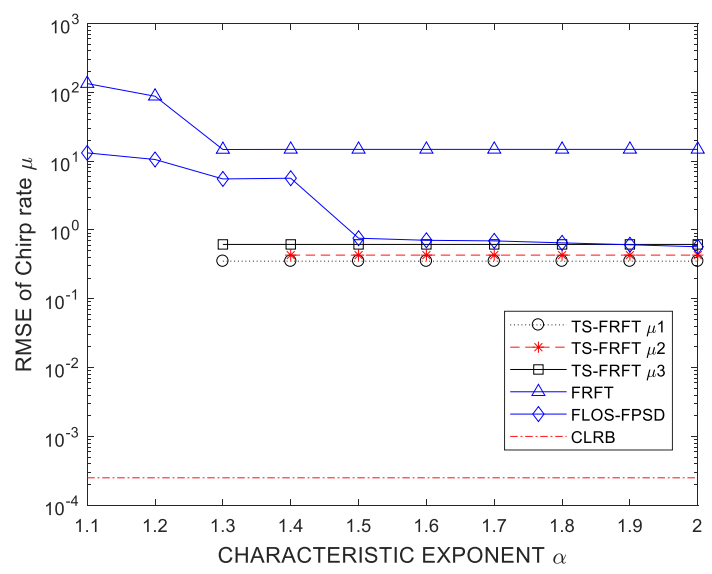

(b)

Fig.4 Estimation accuracy versus Characteristic Exponent. (a) RMSE of initial frequency. (b) RMSE of Chirp rate 
Since the FLOS and tuneable-Sigmoid transform methods can both suppress impulsive noise, the suppression capacity of the FLOS method is insufficient, and the tuneable Sigmoid function suppresses the outliers much harder than the FLOS[21]. Therefore, the estimation performance of the TS-FRFT algorithm is superior to that of FLOS-FPSD algorithm.

Simulation 4: Estimation accuracy with respect to Step size $V_{b}$

In this simulation, the GSNR is set to GSNR $=10 \mathrm{~dB}$ and the characteristic exponent $\alpha$ is set to $\alpha=1.4$. Fig.5 shows the performance versus the step size $V_{b}$ of fractional order $b$. From Fig.5, we can find that the step size directly affects the RMSE of the two parameters. The smaller the fractional step-size, the smaller the RMSE of parameter estimation, but the time complexity will increase.

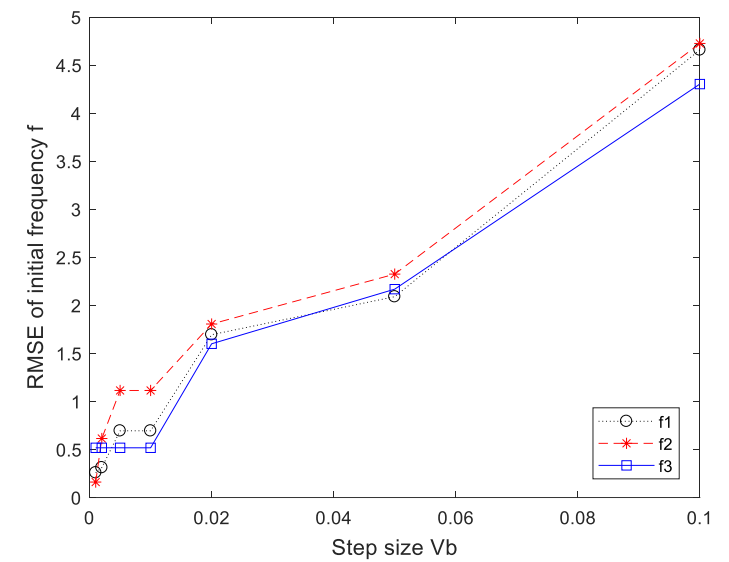

(a)

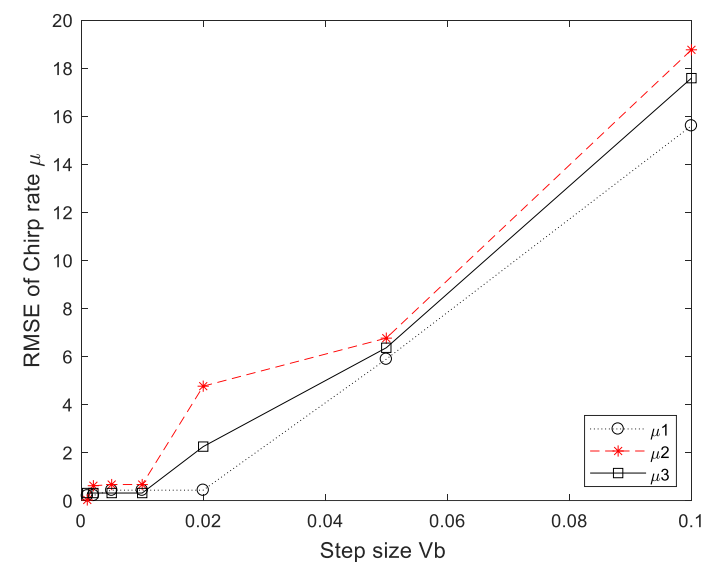

(b)

Fig.5 Estimation accuracy versus step size. (a) RMSE of initial frequency. (b) RMSE of Chirp rate.

\section{Conclusion}

The linear frequency modulated (LFM) signal has been widely implemented in broadband wireless communications in high-speed vehicles, such as internet of vehicles(IoV). In this paper, a novel method, which employs the fractional Fourier transform and the tuneable-Sigmoid transform, is proposed to estimate parameters of multicomponent LFM signals in internet of vehicles(IoV) under the impulsive noise environment. For multicomponent signals, we propose a signal separation technique in the fractional Fourier domain which can effectively suppress the interferences on the detection of the weak components brought by the stronger components through searching peaks, and estimate parameters of LFM signal. Moreover, boundedness and complexity analysis of tuneable-Sigmoid-FFRT to the $S \alpha S$ noise are presented, and the Cramér-Rao bound for parameter estimation is derived and computed in closed form. Both theoretical analysis and simulations demonstrate the superior performances of the proposed approach over other existing methods. Therefore, the proposed method does not need a priori knowledge of noise with higher estimation accuracy under alpha stable distribution noise environment.

\section{Acknowledgments}

We would like to thank the anonymous reviewers for their insightful comments on the paper, as these comments led us to an improvement of the work.

\section{Author information}

\section{Affiliations}


College of Information Engineering, Dalian University, Dalian, Liaoning, China

$\mathrm{Li} \mathrm{Li}$, Mingyan $\mathrm{He}$

Information Science and Technology College, Dalian maritime University, Dalian, Liaoning, China

Xiaofei Shi

Corresponding author

Correspondence to $\mathrm{Li} \mathrm{Li}$.

\section{Authors' contributions}

LL designed the proposed classification algorithms. MH implemented the experiments and completed the analyses of experimental results. XS gave some advice on this manuscript and proofread it. All authors read and approved this submission.

\section{Funding}

This research is supported in part by the Dalian Science and Technology Innovation Fund Project 2020JJ27SN101.

\section{Competing interests}

The authors declare that they have no competing interests.

\section{Availability of data and materials}

Not applicable.

\section{Ethics declarations}

This research does not contain any individual person's data in any form (including individual details, images, or videos).

\section{Abbreviations}

IoV: Internet of Vehicles

LFM: Linear Frequency Modulation

FRFT: Fractional Fourier transform

FLOS: Fractional lower order statistics

TS-FRF: Tuneable Sigmoid fractional Fourier transform

FPSD: Fractional power spectrum function

GSNR: Generalized Signal-Noise-Ratio

PDF: Probability density function

FLOS-FPSD: Fractional power spectrum function based on fractional lower-order statistic

CRB: Cramer-Rao Bound

CRLB: Cramer-Rao Lower Bound

\section{References}

[1] O. Akay, G.F. Boudreaux-Bartels, Fractional convolution and correlation via operator methods and application to detection of liner FM signals. IEEE Trans. Signal Process. 49(5), 979-993 (2001).

[2] S. Z. Qiang, X. Jiang, P. Y. Han, X. Y. Shi, et al, Instantaneous cross-correlation function type of WD based LFM signals analysis via output SNR inequality modeling. EURASIP J. Adv. Signal Process. 122(1), 1-24(2021).

[3] X. Zhang, W. Zhang, Y. Yuan, K. Cui, T. Xie, N. Yuan, DOA estimation of spectrally overlapped LFM signals based on STFT and Hough transform. EURASIP J. Adv. Signal Process. 58(1), 1-10(2019).

[4] H. Gao, J. Li, M. Diao,. Direction finding of bistatic MIMO radar based on quantum-inspired grey wolf 
optimization in the impulse noise. EURASIP J. Adv. Signal Process. 75(12), 1-14(2018).

[5] L. Zheng, C. Yang, C. Yan, H. Qiu, Biorthogonal Fourier transform for multichirp-rate signal detection over dispersive wireless channel. EURASIP J. Wirel. Comm. 23(1), 1-13(2019).

[6] S. Liu, H. H. Zuberi, Y. Lou, M. B. Farooq, S. Shaikh, W. Raza, (2021). M-ary nonlinear sine chirp spread spectrum for underwater acoustic communication based on virtual time-reversal mirror method. EURASIP J. Wirel. Comm. (1), 1-20(2021).

[7] Z. Na, J. Lv, F. Jiang, M. Xiong, N. Zhao, Joint Subcarrier and Subsymbol Allocation-Based Simultaneous Wireless Information and Power Transfer for Multiuser GFDM in IoT, IEEE Internet of Things Journal, 6(4), 5999-6006(2019).

[8] Z. Na, Y. Liu, J. Shi, C. Liu, Z. Gao, UAV-Supported Clustered NOMA for 6G-Enabled Internet of Things: Trajectory Planning and Resource Allocation, IEEE Internet of Things Journal, 8(20), 15041-15048(2021).

[9] Z. Na et al., UAV-Based Wide-Area Internet of Things: An Integrated Deployment Architecture, IEEE Network, 35(5), 122-128(2021).

[10] L. He, K. He, Towards Optimally Efficient Search with Deep Learning for Large-Scale MIMO Systems, IEEE Trans. Commun. 99, 1-14(2022).

[11] L. He K. He, Efficient Memory-Bounded Optimal Detection for GSM-MIMO Systems, IEEE Trans. Commun., 99, 1-15(2022).

[12] A. Serbes, On the Estimation of LFM Signal Parameters: Analytical Formulation, IEEE Transactions on Aerospace and Electronic Systems, 54(2), 848-860 (2018).

[13] O. Aldimashki and A. Serbes, Performance of Chirp Parameter Estimation in the Fractional Fourier Domains and an Algorithm for Fast Chirp-Rate Estimation, IEEE Transactions on Aerospace and Electronic Systems, 56(5), 3685-3700, (2020).

[14] G. Bai, Y. Cheng, W. Tang and S. Li, Chirp Rate Estimation for LFM Signal by Multiple DPT and Weighted Combination, IEEE Signal Process. Lett. 26(1), 149-153, (2019).

[15] J.X. Guo, S.W. Yang, S.W. Qu, J. Hu, Z.P. Nie. A Study on Linear Frequency Modulation Signal Transmission by 4-D Antenna Arrays. IEEE Trans. Antenn. Propag. 63(12), 5409-5416 (2015).

[16] H. Gao, J. Li, M. Diao,. Direction finding of bistatic MIMO radar based on quantum-inspired grey wolf optimization in the impulse noise. EURASIP J. Adv. Signal Process, 75(12), 1-14(2018).

[17] C.L. Nikias, M. Shao. Signal processing with alpha stable distributions and applications. New York: John Wiley \& Sons Inc, 1995. 16.

[18] J.G. Gonzalez, J.L. Paredes, G.R. Arce, Zero-order statistics: A mathematical framework for the processing and characterization of very impulsive signals. IEEE Trans. Signal Process. 54(10), 3839-3851(2006)

[19] Shao, M.; Nikias, C.L. Signal processing with fractional lower order moments: Stable processes and their applications. Proc. IEEE 81, 986-1010(1993).

[20] Y.M. Sun, T.S. Qiu, New HB-weighted time delay estimation algorithm under impulsive noise environment. J Syst Eng Electron. 19(6): 1102-1108 (2008).

[21] L Li,. T.S. Qiu, X.F. Shi, Parameter estimation based on fractional power spectrum density in bistatic MIMO radar system under impulsive noise environment. Circuits Syst. Signal Process. 35(9), 3266-3283(2016).

[22] L.F. Lin, L. Yu, H.Q. Wang, S.C. Zhong. Parameter -adjusted stochastic resonance system for the aperiodic echo chirp signal in optimal FRFT domain. Commun. Nonlinear Sci. Numer. Simul. 43(2), 171-181 (2017).

[23] R. Tao, F. Zhang, Y. Wang. Fractional power spectrum. IEEE Trans. Signal Process. 56(9), 4199-4206 (2008).

[24] T. Qiu, H. Wang, Y. Zhang, H. Bao, Non-linear transform-based robust adaptive latency change estimation of evoked potentials. Methods Archive 41(4), 331-336 (2002).

[25] N. Saini, A. Sinha, Face and palmprint multimodal biometric systems using Gabor-Wigner transform as feature extraction. Pattern Anal. Appl. 18(4), 921-932 (2015).

[26] H. Lang, J. Zhang, X. Zhang, J. Meng, Ship Classification in SAR Image by Joint Feature and Classifier Selection. IEEE Geosci. Remote Sens. Lett. 13(2) 212-216(2016).

[27] K.H. Brodersen, J. Daunizeau, C. Mathys, et.al, Variational Bayesian mixed-effects inference for classification studies. Neuroimage. 76, 345-361(2013).

[28] L. Yu, T.S. Qiu, S.Y. Luan, Robust joint estimation for time delay and Doppler frequency shift based on generalised sigmoid cyclic cross-ambiguity function. IET Radar Sonar Navig. 11(5), 721-728 (2017).

[29] R. Tao, B. Deng, Y. Wang, Research of fractional Fourier transform in signal processing. SCI China Ser. E, 36(1) 113-136(2006).

[30] H.L. Van Trees, Detection, Estimation and Modulation Theory, Part I. New York: wilcy, 1968.

[31] P. Stoica, N. Arye, MUSIC maximum likelihood, and Cramer-Rao bound. IEEE Trans. Acoust., Speech, Signal 
Process. 37(5), 720-741(1989).

[32] A.B. Gershman, M. Pesavento, M.G. Amin, Estimating Parameters of Multiple Wideband Polynomial-phase Sources in sensor arrays. IEEE Trans. Signal Process. 49(2), 2924-2934(2001).

[33] A.L. Swindlehurst, P. Stoica, Maximum Likelihood Methods in Radar Array signal processing. IEEE Proc. 86(2), 421-441 (1998).

[34] I. Bekkerman, J. Tabrikian, Target detection and localization using MIMO radars and sonars. IEEE Trans. Signal Process. 54(10), 3873-3883 (2006).

\section{Figure Title and Legend}

\begin{tabular}{|c|c|c|}
\hline $\begin{array}{l}\text { Figure } \\
\text { Number }\end{array}$ & Title & Detailed Legend \\
\hline Figure 1 & $\begin{array}{l}\text { The spectrum of } \\
\text { FRFT, TS-FRFT } \\
\text { with impulsive } \\
\text { noise (GSNR } \\
=5 \mathrm{~dB} \text { and } \alpha \\
=1.2 \text { ) }\end{array}$ & $\begin{array}{l}\text { (a)The FRFT spectrum of the } \\
\text { LFM signal; } \\
\text { (b) The FRFT spectrum of the } \\
\text { LFM signal with impulsive } \\
\text { noise. } \\
\text { (c) The TS-FRFT spectrum of } \\
\text { the impulsive noise; } \\
\text { (d) The TS-FRFT spectrum of } \\
\text { the multi-component LFM } \\
\text { signals with impulsive noise }\end{array}$ \\
\hline Figure 2 & $\begin{array}{l}\text { The spectrum of } \\
\text { FRFT and TS- } \\
\text { FRFT under the } \\
\text { SaS noise }\end{array}$ & $\begin{array}{l}\text { (a) The FRFT spectrum of the } \\
\text { LFM signals without noise and } \\
\text { its rotation angle and frequency } \\
\text { section planes. } \\
\text { (b) The FRFT spectrum of the } \\
\text { LFM signals with impulsive } \\
\text { noise and its rotation angle and } \\
\text { frequency section planes. } \\
\text { (c)The tuneable-Sigmoid-FRFT } \\
\text { spectrum of the LFM signals } \\
\text { with impulsive noise and its } \\
\text { rotation angle frequency section } \\
\text { planes }\end{array}$ \\
\hline Figure 3 & $\begin{array}{l}\text { Estimation } \\
\text { accuracy versus } \\
\text { GSNR }\end{array}$ & $\begin{array}{l}\text { (a) RMSE of initial frequency. } \\
\text { (b) RMSE of Chirp rate. }\end{array}$ \\
\hline Figure 4 & $\begin{array}{l}\text { Estimation } \\
\text { accuracy versus } \\
\text { Characteristic } \\
\text { Exponent }\end{array}$ & $\begin{array}{l}\text { (a) RMSE of initial frequency. } \\
\text { (b) RMSE of Chirp rate }\end{array}$ \\
\hline Figure 5 & $\begin{array}{l}\text { Estimation } \\
\text { accuracy versus } \\
\text { step size }\end{array}$ & $\begin{array}{l}\text { (a) RMSE of initial frequency. } \\
\text { (b) RMSE of Chirp rate }\end{array}$ \\
\hline
\end{tabular}

